\title{
Erste Studie zu jungen Frauen mit Flucht- erfahrung in Deutschland veröffentlicht
}

Kaum eine Gruppe mit Fluchterfahrung ist so verletzlich wie Mädchen und junge Frauen. Save the Children veröffentlicht dazu gemeinsam mit der Charité - Universitätsmedizin Berlin die bundesweit erste Bedarfsanalyse aus deutschen Erstaufnahmeeinrichtungen. Vor allem die Gefahren auf der Flucht liegen für junge Frauen deutlich höher. Schnell werden sie zur Zielscheibe von sexualisierter Gewalt, Ausbeutung und gesellschaftlicher Benachteiligung. Ihre psychosoziale Entwicklung ist durch traumatische Erlebnisse besonders gefährdet. Die Studie zeigt, dass auch in Deutschland entsprechende Schutzstrukturen zur Erholung und Aufarbeitung von Belastungssituationen fehlen. Viele der Mädchen leben isoliert und haben wenig Zugang zu unterstützenden Angeboten und sozialer Teilhabe. dung Charité, Berlin 11.07.2019
Quelle: Presseaussen-

Aus der Studie lässt sich ableiten, dass die Selbstwirksamkeit und die Resilienz der Mädchen und jungen Frauen durch psychosoziale Förderangebote bewusst gestärkt werden müssen.„Eine Stärkung junger Frauen von innen heraus ist uns ein großes Anliegen. Ihre Förderung trägt letztendlich zum Wohl der ganzen Gesellschaft bei", resümiert RubyRebecca Brinza, Fachliche Leitung Migration und Flucht von Save the Children Deutschland. Die Studie zeigt einen speziellen Bedarf nach genderspezifischer Förderung bei Mädchen und jungen Frauen auf, deren innere Stärkung ein wichtiges Anliegen des Projektes "Mädchen.Machen.Mut." ist. Prof. Meryam Schouler-Ocak, Professorin für Interkulturelle Psychiatrie und Psychotherapie an der Charité, fasst zusammen: „Mütter und Kinder

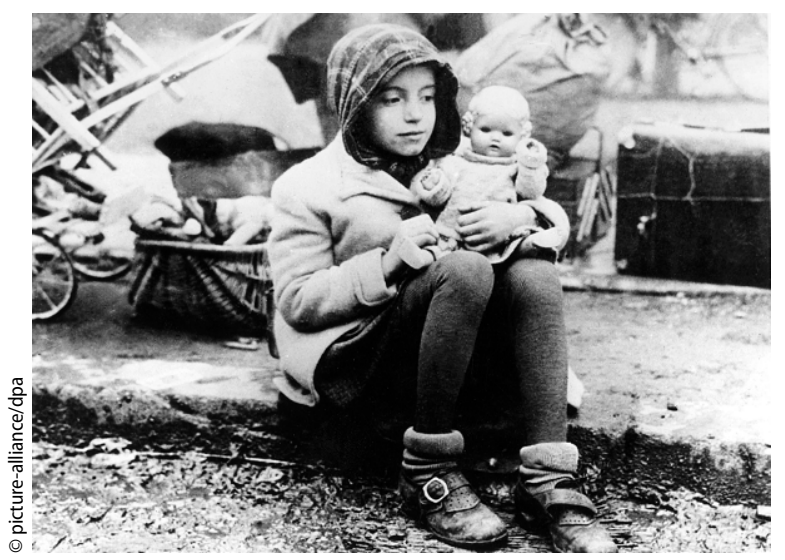

auf der Flucht sind unglaublichem Leid ausgesetzt. Zwangsprostitution, Hunger und Tod naher Verwandter müssen aufgearbeitet werden, damit eine Traumafolgestörung verhindert werden und die Integration gelingen kann."

Zugleich verfügen junge Mädchen und Frauen über individuelle Ressourcen, die es zu stärken gilt. Kreativität, soziale Einbindung, Selbstwertgefühl und das Erleben von Selbstwirksamkeit fördern die Verarbeitung belastender Erlebnisse und ein generelles Wohlbefinden. In der Analyse benannten die Mädchen selbst kreative, musische, handwerkliche und naturpädagogisch ausgerichtete Angebote als unterstützend und wichtig. Nonverbale Kommunikation ermöglicht dabei über Sprachbarrieren hinweg einen Austausch in der Gruppe. Gleichzeitig können schöpferische Tätigkeiten sowohl den emotionalen Ausdruck als auch das Erleben von Handlungsfähigkeit fördern. Den Mädchen soll ermöglicht werden, sich in geschlechtsspezifischen, psychosozialen Gruppenangeboten sicher zu fühlen, soziale Beziehungen zu knüpfen und sich altersangemessen und spielerisch mitzuteilen.

Heranwachsende mit Fluchterfahrung brauchen eine altersgerechte Unterbringung, die Regelbeschulung und auf sie zugeschnittene Angebote.
Dazu gehören die psychosoziale Unterstützung und Förderung der Selbstwirksamkeit, altersgerechte Angebote zur entwicklungspsychologischen Förderung und Kontakt zu Gleichaltrigen sowie Bildungsangebote und bedarfsgerechte Lernmöglichkeiten. Zudem benötigen die Mädchen und junge Frauen Schutz durch Gender- und kultursensible Gewaltschutzkonzepte, sie sollen gesellschaftlich beteiligt werden und Möglichkeit zur Mitbestimmung, beispielsweise bei der Essensversorgung, bekommen. Darüber hinaus bedarf es einer umfassenden und bedarfsgerechten Gesundheitsversorgung.

Das Projekt „Mädchen.Machen. Mut." von Save the Children Deutschland e. V. wird von der Cummins Foundation gefördert.

\section{Weitere Informationen:}

https://www.savethechildren.de/ http://www.charite.de

Paediatr. Paedolog. 2019 · 54:248 https://doi.org/10.1007/s00608019-00716-w (c) Springer-Verlag GmbH Austria, ein Teil von Springer Nature 2019 